\title{
Padrão de deslocamento ativo da região metropolitana de Campinas, Brasil
}

\section{Campinas metropolitan region's active commuting pattern}

\section{AUTORES \\ Guilherme Stefano Goulardins ${ }^{1}$ (D) \\ Thiago Hérick de Sá2 (D) \\ Eduardo Kokubun ${ }^{1}$ iD \\ 1 Universidade Estadual Paulista "Júlio de Mesquita Filho", Departamento de Educação Física, Rio \\ Claro, São Paulo, Brasil. \\ 2 Universidade de São Paulo, Núcleo de Pesquisas Epidemiológicas em Nutrição e Saúde, São Paulo, São Paulo, Brasil.}

\section{CONTATO}

Guilherme Stefano Goulardins

guigoulardins@gmail.com

Avenida 24 A,1515 - Bela Vista - Rio Claro, São Paulo, Brasil.

CEP: $13506-900$.

DOI

$10.12820 /$ rbafs.26e0202

\section{(cc) BY}

Este trabalho está licenciado com uma Licença Creative Commons - Atribuição 4.0 Internacional.

\begin{abstract}
RESUMO
Analisar o padrão do deslocamento ativo da Região Metropolitana Campinas, levando em consideração particularidades dos residentes e suas viagens. Utilizando as bases de dados obtidas através da Pesquisa Origem e Destino da Região Metropolitana de Campinas dos anos de 2003 e 2011, foram realizadas estatísticas descritivas e temporais a partir das características dos sujeitos, municípios e viagens. Para identificarmos diferenças adotamos o intervalo de confiança de 95\% (IC 95\%). Utilizamos a regressão de Poisson para verificação da correlação entre características individuais e o desfecho ser ciclista ou caminhante, adotando o valor de $\mathrm{p}<0,05$. Todos os dados foram analisados levando-se em consideração os procedimentos de amostragem para que a amostra fosse representativa. Constatou-se que a frequência de ciclistas e caminhantes e suas respectivas viagens caíram. Encontramos associação positiva para ciclista ser homem e classes econômicas mais baixas, para os caminhantes houve associação positiva ser mulher e crianças/adolescentes. Quanto ao tempo de viagem, notou-se aumento na mediana para os ciclistas e queda para os caminhantes. Não encontramos nenhuma viagem de bicicleta que faça integração com outro modo de transporte. Quanto ao porte do município, observou-se uma queda em viagens de bicicleta tanto nos municípios pequenos, médios e grandes, e os residentes de Campinas apresentaram as menores frequências.
\end{abstract}

Palavras-chave: Ciclistas; Caminhantes; Bicicleta; Caminhada; Transporte.

\section{ABSTRACT}

This study analyzes the active commuting pattern of the Metropolitan Region of the City of Campinas, Brazil, by considering its commuters and their features. By using the database of Pesquisa Origem e Destino (a Brazilian instrument for transportation planning) of Campinas Metropolitan Region 2003-2011, we present descriptive and temporal statistics concerning the characteristics of the region's commuters, cities, and journeys. To assess those, we adopted a confidence interval of 95\% (CI 95\%). We used the Poisson regression to check the correlation between individual characteristics and outcomes of being a cyclist or a pedestrian, adopting the value of $p<0.05$. We took into consideration the procedures for datum obtaining to present representative samples, verifying that the number of both cyclists and pedestrians has dropped. We found positive associations regarding low-income males and being a cyclist; as well as females and children/teenagers and being pedestrians. The mean length of commuting time has increased for cycling journeys and decreased for pedestrian ones. We could not find a bicycle journey that integrates with another mean of transportation. Regarding the size of a city, we observed that the number of cycling journeys has dropped in small, medium, and large municipalities; Campinas presents the lowest index.

Keywords: Cyclists; Pedestrians; Bicycle; Walking; Transportation.

\section{Introdução}

O deslocamento ativo, além de apresentar grande diversidade quanto a sua classificação e denominação, também possui múltiplos modos de realização, como patins, patinetes, canoas e os mais comuns, a bicicleta e a caminhada ${ }^{1}$. Para o presente estudo, define-se deslocamento ativo como todo modo de transporte movido a propulsão humana ${ }^{2}$.

Essa forma de deslocamento proporciona benefícios individuais, como: fator de proteção para doenças cardiovasculares $^{3}$ e doenças crônicas não transmissí- veis, redução de obesidade e mortalidade ${ }^{4,5}$, quanto coletivos, tendo como exemplo a diminuição de poluição sonora e atmosférica e redução de congestionamentos e uso de combustíveis fosseis ${ }^{1,6}$.

Dentre os modos de deslocamento ativo, a caminhada é a mais frequente ${ }^{7}$ no Brasil, posiciona-se como segundo principal modo de transporte da população (24\%), e a bicicleta ocupa o $4^{\circ}$ lugar $(8 \%)^{8}$, a qual apresenta baixa frequência se comparada a países europeus como Dinamarca e Países Baixo ${ }^{9}$. Tratando-se da caminhada, verifica-se valores superiores à média da 
América Latina e Caribe ${ }^{10}$.

No contexto brasileiro, diversos são os fatores associados ao deslocamento ativo, sendo eles individuais/ pessoais, ambientais, psicossociais, além de características próprias de cada deslocamento ${ }^{11,12}$. Para a bicicleta, os fatores positivamente associados são: ser do sexo masculino, atingir o nível de atividade física recomendado, dispor de ciclovias/ciclofaixas, ter bicicleta, ser residente de centros urbanos, maior densidade das ruas e a proximidade do destino $(<4 \mathrm{~km})^{11}$. Já para a caminhada, os fatores positivamente associados se relacionam ao sexo feminino, não possuir carro e morar em bairros mais vulneráveis ${ }^{13}$.

Constata-se também uma variação de sua frequência entre as cidades no Brasi ${ }^{10}$, visto que as interioranas possuem frequências maiores que capitais e grandes municípios. Como levantado por Bauman et al. ${ }^{14}$, é necessário estudar diferentes regiões para compreendermos as características ambientais, individuais e peculiares de cada tipo de deslocamento. Com isso, analisar a Região Metropolitana de Campinas faz-se importante diante das diferentes características de seus municípios e a carência de estudos sobre o tema na região.

Nesse sentido, o objetivo do estudo é analisar o padrão do deslocamento ativo da Região Metropolitana de Campinas, levando em consideração a frequência, fatores individuas e as diferentes características dos municípios, como dimensões territoriais e características sociodemográficas.

\section{Métodos}

A Região Metropolitana de Campinas está localizada a noroeste da capital do Estado de São Paulo e atualmente conta com 20 munícipios e possui atualmente renda domiciliar mensal per-capita de $\mathrm{R} \$ 1.151,15$ e Índice de Desenvolvimento Humano (IDH) de 0,792, entretanto, nos anos de 2003 e 2011, possuía 19 municípios. É a segunda maior região metropolitana do estado de São Paulo em população, no ano de 2003 sua população era de 2,3 milhões de habitantes, passando para aproximadamente 2,7 milhões de habitantes no ano de 2011, a densidade demográfica no ano de 2003 era de $626,2 \mathrm{hab} / \mathrm{km}^{2}$ e passando para $761,5 \mathrm{hab} / \mathrm{km}^{2} \mathrm{e}$ sua área total era de $3.673 \mathrm{~km}^{2} 15$.

Foram empregados dados da Pesquisa de Origem e Destino da Região Metropolitana de Campinas, São Paulo realizada nos anos de 2003 e 2011, as únicas realizadas nessa região e sem previsão de futuras pesquisas acerca desse tema. Os dados da pesquisa Origem e
Destino de 2003 foram utilizados para as análises de tendência temporal, já a pesquisa de 2011 foi o foco do presente estudo e a principal base de dados. Essa pesquisa é dividida em: Pesquisa linha de contorno que são viagens externas a Região Metropolitana de Campinas e Pesquisa domiciliar, referente as viagens internas da Região Metropolitana de Campinas.

A pesquisa Origem e Destino foi realizada em 4 etapas: Planejamento, trabalhos de campo, sistematização das informações e síntese dos resultados. Essas etapas são descritas mais detalhadamente no estudo de Goulardins ${ }^{15}$, no entanto, de forma resumida, contaram com o levantamento e sistematização das informações, preparação das zonas de pesquisa, elaboração dos questionários e definição da amostragem mínima dos domicílios a serem pesquisados. Seguidamente, foi executado o arrolamento dos domicílios à aplicação dos questionários, treinamento dos entrevistadores e a aplicação dos questionários. Por fim, foi realizado o controle e verificação das informações coletadas.

As taxas brutas dos ciclistas e caminhantes (por 1000 habitantes) foram estimadas segundo características individuais para o ano de 2011. Para identificarmos diferenças, adotamos o intervalo de confiança de 95\% (IC 95\%). Utilizamos a regressão de Poisson para verificarmos a correlação entre características individuais e o desfecho ciclista ou caminhante, além do intervalo de confiança 95\%, adotou-se o valor de $p<$ 0,05 . Foi realizada dessa maneira para fortalecermos a análise estatística.

Ademais, estimamos a frequência (adotamos esse termo assim como $\mathrm{Sá}^{2}$, uma vez que prevalência normalmente remete a doenças e comportamentos) de ciclistas e caminhantes e suas respectivas viagens desses modos de transporte nos anos de 2003 e 2011, adotando o IC 95\%. Levantamos também o tempo de duração das viagens da bicicleta e caminhada, que foram submetidos ao teste Kolmogorov-Smirnov a fim de verificar a normalidade dos dados.

Todos os dados foram analisados levando-se em consideração os procedimentos de amostragem para ajustar as respostas que foram coletadas a nível individual para que a amostra fosse representativa da área estudada. Essas análises foram realizadas utilizando o Stata 12.0.

Existem diversos modelos teóricos e os mesmos nos permitem compreender o porquê e como os indivíduos adotam determinado comportamento ${ }^{16}$ e, no âmbito da atividade física, encontramos diversos modelos e teorias ${ }^{17}$.

A atividade física, assim como outros comporta- 
mentos, envolve uma série de fatores que a torna um fenômeno complexo ${ }^{17}$. Outro problema dos modelos em atividade física é que a maioria deles focam em investigar os fatores individuais ${ }^{17}$, dificultando áreas que estudam esse comportamento em grandes populações.

Diante disso adotamos o modelo proposto por $\mathrm{Du}^{-}$ mith $^{17}$, que consiste em três níveis de determinação causal, sendo eles determinantes distais (fatores demográficos, ex: sexo, idade, cor da pele), intermediários (fatores socioeconômicos, ex: renda, escolaridade, ocupação) e proximais (fatores comportamentais, ex: tabagismo, dieta, consumo de álcool). Nesse modelo todos os níveis citados correlacionam com o desfecho, podendo ser realizada de forma global.

O modelo final utilizado no presente estudo não possui os determinantes proximais, que são os fatores comportamentais já que não foram coletados na pesquisa Origem e Destino. Dessa forma, no primeiro nível (distais) encontram-se os fatores demográficos sexo e idade, no segundo nível (intermediários) situam-se os fatores socioeconômicos grau de instrução, renda e condição da atividade, e o desfecho era ser ciclista ou caminhante.

Esse modelo serviu de base para investigar a correlação levantada anteriormente e realizar a Regressão de Poisson.

\section{Resultados}

A amostra foi constituída por 36.892 pessoas residentes da Região Metropolitana de Campinas e seus respectivos dados sociodemográficos. Vale salientar que esses dados remetem ao ano de 2011, uma vez que as análises sobre as características sociodemográficas remetem apenas a esse ano.

Na Tabela 1, observamos que 51,6\% (IC95\%: 51,1 - 52,1) de mulheres, 35,7\% (IC95\%: 35,2 - 36,2) de adultos jovens, sobre o grau de instrução, $41,7 \%$ (IC95\%: 41,2 - 42,3) de pessoas possuem o fundamental I/II completo ou incompleto. Em relação aos aspectos socioeconômicos, pontuamos que $61,4 \%$ (IC95\%: 60,3 - 62,5) se encontram na classe E, outros $44,6 \%$ (IC 95\%: 44,0 - 45,1) declararam estar ocupados na condição de atividade trabalhista.

Entre 2003 e 2011 (Tabela 2) houve uma queda significante na frequência de ciclistas na Região Metropolitana de Campinas, passando de 2,2 (IC95\%: 2,0 - 2,3) no ano de 2003 para 1,6 (IC95\%: 1,4 - 1,7) em 2011, assim como suas viagens que eram de 3,5\% (IC95\%: 3,2 - 3,8) em 2003 e decaíram para 2,1\% (IC95\%: 2,0 - 2,2) em 2011.
Tabela 1 - Tabela descritiva da população da Região Metropolitana de Campinas (RMC) nos anos de 2003 e 2011 de acordo com as características sociodemográficas

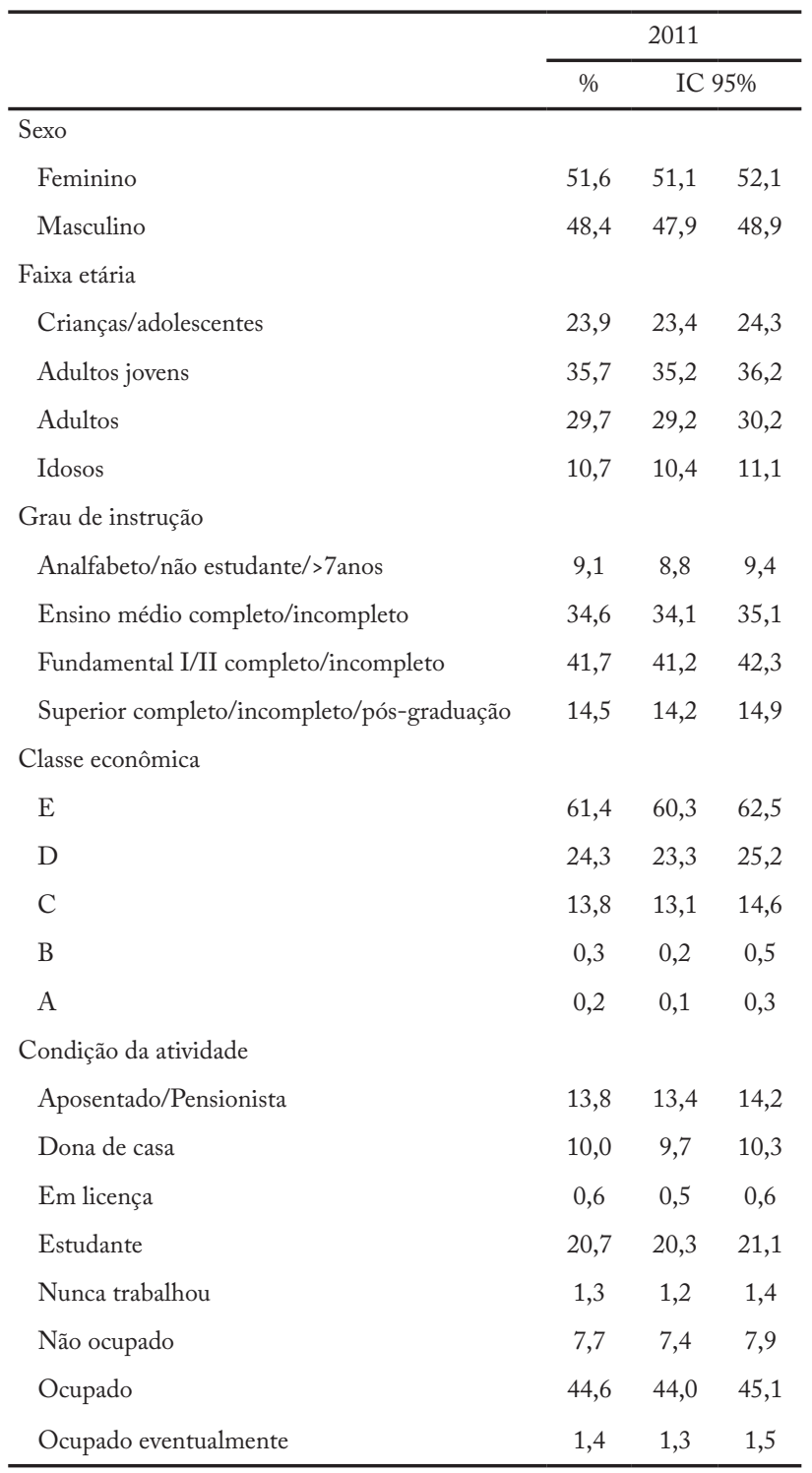

*n = 36.892 (2011); IC 95\% = Intervalo de Confiança 95\%. *Diferenças metodológicas entre os anos de coleta.

Observa-se também uma redução significante na frequência de caminhantes: no ano de 2003 era de 21,9\% (IC95\%: 21,4-22,4) passando para 17,3\% (IC95\%:16,9 - 17,7) em 2011, tal como suas viagens que em 2003 contribuíam com 35,4\% (IC95\%: 34,7 - 36,1) sendo reduzidas a 24,0\% (IC95\%: 23,7 - 24,4) em 2011.

Em relação ao tempo das viagens, apresentaremos os mesmos em forma de mediana, já que os dados não possuem uma distribuição normal. Entre 2003 e 2011 pudemos observar um aumento no tempo de viagem para bicicletas que era de 15 minutos (intervalo interquartil de 12,5 minutos) em 2003 e passou para 20 mi- 
nutos (intervalo interquartil de 20 minutos) em 2011 e uma queda para as viagens a pé que era de $20 \mathrm{minu}-$ tos (intervalo interquartil de 20 minutos) em 2003 e passando para 15 minutos (intervalo interquartil de 10 minutos) em 2011. Ressalta-se que não foi encontrada nenhuma viagem de bicicleta que tenha feito integração com outros modais.

Quando analisamos os ciclistas e caminhantes segundo municípios da Região Metropolitana de Campinas (Tabela 3), verificamos que em ambos, todas as categorias de município apresentaram redução na frequência de seus residentes e os de Campinas tiveram as frequências mais baixas em relação aos outros municípios.

Tabela 2 - Frequência dos ciclistas e caminhantes e suas viagens na Região Metropolitana de Campinas e frequência dos ciclistas e caminhantes segundo a classificação do município nos anos de 2003 e 2011

\begin{tabular}{|c|c|c|c|c|c|c|}
\hline \multirow{3}{*}{$\begin{array}{l}\text { Grupos modos de } \\
\text { transporte }\end{array}$} & \multicolumn{3}{|c|}{2003} & \multicolumn{3}{|c|}{2011} \\
\hline & \multirow{2}{*}{$\begin{array}{c}\% \\
2,2\end{array}$} & \multicolumn{2}{|c|}{ IC 95\% } & \multirow{2}{*}{$\begin{array}{c}\% \\
1,6\end{array}$} & \multicolumn{2}{|c|}{ IC 95\% } \\
\hline & & 2,0 & 2,3 & & 1,4 & 1,7 \\
\hline Caminhantes & 21,9 & 21,4 & 22,4 & 17,3 & 16,9 & 17,7 \\
\hline Viagens/bicicleta & 3,5 & 3,2 & 3,8 & 2,1 & 2,0 & 2,2 \\
\hline Viagens/a pé & 35,4 & 34,7 & 36,1 & 24,0 & 23,7 & 24,4 \\
\hline \multirow{3}{*}{ Grupos de município } & \multicolumn{6}{|c|}{ Ciclistas } \\
\hline & \multicolumn{3}{|c|}{2003} & \multicolumn{3}{|c|}{2011} \\
\hline & $\%$ & \multicolumn{2}{|c|}{ IC 95\% } & $\%$ & \multicolumn{2}{|c|}{ IC 95\% } \\
\hline$<100.000$ habitantes & 6,5 & 5,9 & 7,0 & 4,4 & 3,9 & 4,8 \\
\hline$>100.000$ habitantes & 4,9 & 4,6 & 5,3 & 2,8 & 2,6 & 3,0 \\
\hline Campinas & 0,9 & 0,8 & 1,0 & 0,7 & 0,6 & 0,8 \\
\hline \multirow{3}{*}{ Grupos de município } & \multicolumn{6}{|c|}{ Caminhantes } \\
\hline & \multicolumn{3}{|c|}{2003} & \multicolumn{3}{|c|}{2011} \\
\hline & $\%$ & \multicolumn{2}{|c|}{ IC 95\% } & $\%$ & \multicolumn{2}{|c|}{ IC 95\% } \\
\hline$<100.000$ habitantes & 37,9 & 36,8 & 39,0 & 27,2 & 26,3 & 28,1 \\
\hline$>100.000$ habitantes & 40,9 & 40,1 & 41,6 & 28,8 & 28,2 & 29,3 \\
\hline Campinas & 30,2 & 29,6 & 30,9 & 19,2 & 18,8 & 19,7 \\
\hline
\end{tabular}

${ }^{*} \mathrm{n}=26.064 ;$ IC 95\% = Intervalo de Confiança 95\%.

Podemos observar na Tabela 3, que, para os ciclistas, há maior frequência de homens em relação às mulheres, dado que a cada 1000 habitantes da Região Metropolitana de Campinas, 25,1\% são do sexo masculino, para idade, a maior frequência foi dos adultos jovens $(21,1 \%)$. Em relação a classe econômica, nota -se o predomínio da classe E $(29,8 \%)$ e ausência das mais elevadas (A/B). Para o grau de instrução o domínio foi a categoria ensino médio completo/incompleto $(19,9 \%)$ e para a condição da atividade, aqueles que se disseram ocupados eventualmente $(29,8 \%)$ foram os mais frequentes.
Tabela 3 - Características sociodemográficas dos ciclistas e caminhantes da Região Metropolitana de Campinas no ano de 2011

\begin{tabular}{|c|c|c|c|c|c|c|}
\hline & \multicolumn{3}{|c|}{$\begin{array}{c}\text { Ciclistas } \\
\text { (por } 1000 \text { habitantes) }\end{array}$} & \multicolumn{3}{|c|}{$\begin{array}{c}\text { Caminhantes } \\
\text { (por } 1000 \text { habitantes) }\end{array}$} \\
\hline & $\%$ & \multicolumn{2}{|c|}{ IC 95\% } & $\%$ & \multicolumn{2}{|c|}{ IC 95\% } \\
\hline \multicolumn{7}{|l|}{ Sexo } \\
\hline Feminino & 6,6 & 5,3 & 7,9 & 184,4 & 178,6 & 190,2 \\
\hline Masculino & 25,1 & 22,6 & 27,6 & 160,2 & 154,6 & 165,9 \\
\hline \multicolumn{7}{|l|}{ Idade } \\
\hline Crianças/adolescentes & 11,5 & 9,0 & 14,0 & 367,4 & 356,8 & 378,0 \\
\hline Adultos jovens & 21,1 & 18,4 & 23,8 & 116,8 & 111,0 & 122,6 \\
\hline Adultos & 16,0 & 13,4 & 18,5 & 110,0 & 103,9 & 116,1 \\
\hline Idosos & 5,1 & 2,7 & 7,6 & 99,0 & 89,4 & 108,7 \\
\hline \multicolumn{7}{|l|}{ Classe econômica } \\
\hline $\mathrm{E}$ & 29,8 & 24,7 & 34,9 & 207,9 & 196 & 219,7 \\
\hline $\mathrm{D}$ & 17,0 & 11,0 & 23,0 & 100,0 & 86,4 & 113,6 \\
\hline $\mathrm{C}$ & 9,3 & 3,4 & 15,2 & 82,5 & 66,7 & 98,3 \\
\hline B & 0,0 & 0,0 & 0,0 & 31,3 & 0,0 & 91,7 \\
\hline A & 0,0 & 0,0 & 0,0 & 63,9 & 0,0 & 184,4 \\
\hline \multicolumn{7}{|l|}{ Grau de instrução } \\
\hline $\begin{array}{l}\text { Analfabeto/não } \\
\text { estudante/>7anos }\end{array}$ & 6,0 & 3,1 & 8,9 & 155,2 & 142,2 & 168,1 \\
\hline $\begin{array}{l}\text { Ensino médio completo/ } \\
\text { incompleto }\end{array}$ & 19,9 & 17,2 & 22,6 & 154,3 & 147,7 & 160,9 \\
\hline $\begin{array}{l}\text { Fundamental I/II } \\
\text { completo/incompleto }\end{array}$ & 17,4 & 15,2 & 19,6 & 224,9 & 217,9 & 231,8 \\
\hline $\begin{array}{l}\text { Superior completo/ } \\
\text { incompleto/pós-graduação }\end{array}$ & 5,9 & 3,8 & 8,1 & 77,6 & 70,4 & 84,8 \\
\hline \multicolumn{7}{|l|}{ Condição da atividade } \\
\hline Aposentado/Pensionista & 5,8 & 3,5 & 8,2 & 90,5 & 82,3 & 98,7 \\
\hline Dona de casa & 1,7 & 0,2 & 3,1 & 133,0 & 121,2 & 144,7 \\
\hline Em licença & 16,6 & 0,0 & 39,4 & 72,8 & 36,7 & 108,8 \\
\hline Estudante & 10,4 & 7,9 & 12,9 & 413,4 & 401,8 & 425,0 \\
\hline Nunca trabalhou & 2,0 & 0,0 & 5,9 & 19,1 & 7,2 & 31,1 \\
\hline Não ocupado & 7,3 & 3,6 & 10,9 & 62,4 & 52,8 & 72,0 \\
\hline Ocupado & 25,5 & 22,9 & 28,1 & 119,4 & 114,2 & 124,6 \\
\hline Ocupado eventualmente & 29,8 & 14,4 & 45,2 & 194,2 & 158,5 & 229,9 \\
\hline
\end{tabular}

${ }^{*} \mathrm{n}=26.064 ;$ IC 95\% = Intervalo de Confiança 95\%.

Para os caminhantes, as mulheres $(184,4 \%)$ foram mais frequentes, para idade, as crianças e adolescentes $(367,4 \%)$ foram os mais frequentes em relação a adultos e idosos. Assim como observado para os ciclistas, a classe econômica E $(207,9 \%)$ é a mais recorrente. Com relação a condição da atividade e grau de instrução, os mais frequentes foram estudantes $(413,4 \%)$ e aqueles com Fundamental I/II Completo/incompleto (224,9\%), respectivamente.

Nas Tabelas 4 e 5 são apresentadas as análises de 
regressão de Poisson (brutas e ajustadas), através das quais notou-se que as variáveis associadas ao desfecho de ser ciclista, foram sexo masculino e classe econômica mais baixa. Já para os caminhantes constatou-se que a associação ao desfecho foram as variáveis sexo feminino e faixa etária de crianças e adolescentes, cujas variáveis apresentam o valor de $\mathrm{p}<0,05$.

\section{Discussão}

A partir das análises dos resultados, encontramos uma queda na frequência de ciclistas e caminhantes e suas respectivas viagens. Tal fenômeno não se restringe apenas a Região Metropolitana de Campinas, já que também é observado em outros locais do Brasil, como em
São Paulo ${ }^{18}$. O declínio pode ser resultado das estratégias adotadas pelo governo federal em 2007 a fim de combater a crise econômica, que facilitou os empréstimos e realizou uma política de isenção fiscal para alguns produtos, incluindo os automóveis ${ }^{19}$. Aliado a isso, no estado de São Paulo, a frota de veículos aumentou 156\% no período entre 2001 e 2018, na Região Metropolitana de Campinas teve um aumento de $165 \%{ }^{20}$.

No que diz respeito aos fatores associados, obtivemos que para os ciclistas as características que tiveram associação positiva foram sexo masculino e classe econômica mais baixa. Em relação ao sexo encontramos que homens tem aproximadamente 3 vezes a chance de ser ciclistas das mulheres, que apesar de alto, foi in-

Tabela 4- Regressão de Poisson dos desfechos dos ciclistas com as variáveis independentes sexo, idade, classe econômica, grau de instrução e condição da atividade.

\begin{tabular}{|c|c|c|c|c|c|c|c|c|}
\hline \multirow{2}{*}{$\begin{array}{ll} & \text { Ciclistas } \\
\text { Sexo }\end{array}$} & \multirow[t]{2}{*}{ RP Bruta } & \multicolumn{2}{|c|}{ IC 95\% } & \multirow[t]{2}{*}{$\mathrm{p}$} & \multirow[t]{2}{*}{ RP Ajustada } & \multicolumn{2}{|c|}{ IC $95 \%$} & \multirow[t]{2}{*}{$\mathrm{p}$} \\
\hline & & & & & & & & \\
\hline Feminino & Ref & Ref & Ref & & Ref & Ref & Ref & \\
\hline Masculino & 3,27 & 2,63 & 4,06 & 0,001 & 4,20 & 2,49 & 7,07 & 0,001 \\
\hline \multicolumn{9}{|l|}{ Idade } \\
\hline Crianças/adolescentes ( 0 - 18 anos) & Ref & Ref & Ref & & Ref & Ref & Ref & \\
\hline Adultos jovens (19-40 anos) & 1,88 & 1,47 & 2,42 & 0,001 & 1,96 & 0,19 & 20,24 & 0,572 \\
\hline Adultos (41 - 64 anos) & 1,72 & 1,32 & 2,25 & 0,001 & 1,68 & 0,16 & 17,74 & 0,665 \\
\hline Idosos (> 65 anos) & 0,90 & 0,53 & 1,52 & 0,695 & 0,73 & 0,06 & 8,59 & 0,805 \\
\hline \multicolumn{9}{|l|}{ Classe econômica } \\
\hline $\mathrm{E}$ & Ref & Ref & Ref & & Ref & Ref & Ref & \\
\hline $\mathrm{D}$ & 0,54 & 0,36 & 0,80 & 0,002 & 0,45 & 0,30 & 0,67 & 0,001 \\
\hline $\mathrm{C}$ & 0,30 & 0,15 & 0,57 & 0,001 & 0,32 & 0,16 & 0,65 & 0,002 \\
\hline $\mathrm{B}$ & 0,00 & 0,00 & 0,00 & 0,001 & 0,00 & 0,00 & 0,00 & 0,001 \\
\hline A & 0,00 & 0,00 & 0,00 & 0,001 & 0,00 & 0,00 & 0,00 & 0,001 \\
\hline \multicolumn{9}{|l|}{ Grau de instrução } \\
\hline Analfabeto/não estudante/>7anos & Ref & Ref & Ref & & Ref & Ref & Ref & \\
\hline Ensino médio completo/incompleto & 2,08 & 1,26 & 3,42 & 0,004 & 0,61 & 0,27 & 1,36 & 0,224 \\
\hline Fundamental I/II Completo/incompleto & 2,09 & 1,27 & 3,43 & 0,004 & 0,85 & 0,39 & 1,84 & 0,675 \\
\hline Superior completo/incompleto/pós-graduação & 0,57 & 0,31 & 1,03 & 0,063 & 0,23 & 0,08 & 0,68 & 0,008 \\
\hline \multicolumn{9}{|l|}{ Condição da atividade } \\
\hline Aposentado/Pensionista & Ref & Ref & Ref & & Ref & Ref & Ref & \\
\hline Dona de casa & 0,30 & 0,12 & 0,78 & 0,013 & 0,22 & 0,03 & 1,68 & 0,144 \\
\hline Em licença & 2,51 & 0,61 & 10,24 & 0,200 & 1,79 & 0,27 & 11,65 & 0,544 \\
\hline Estudante & 0,72 & 0,45 & 1,15 & 0,169 & 0,99 & 0,12 & 7,86 & 0,989 \\
\hline Nunca trabalhou & 1,47 & 0,20 & 10,69 & 0,701 & 0,00 & 0,00 & 0,00 & 0,001 \\
\hline Não ocupado & 1,90 & 1,00 & 3,60 & 0,049 & 1,59 & 0,69 & 3,66 & 0,273 \\
\hline Ocupado & 1,83 & 1,21 & 2,77 & 0,004 & 0,90 & 0,49 & 1,67 & 0,738 \\
\hline Ocupado eventualmente & 2,83 & 1,47 & 5,42 & 0,002 & 1,36 & 0,55 & 3,40 & 0,509 \\
\hline
\end{tabular}

*n = 26.064; Significância estatística se p < 0,05; RP ajustada segundo o modelo conceitual, incluindo sexo, idade, classe econômica, grau de instrução e condição da atividade; IC 95\% = Intervalo de Confiança 95\%. 
Tabela 5 - Regressão de Poisson dos desfechos dos caminhantes com as variáveis independentes sexo, idade, classe econômica, grau de instrução e condição da atividade.

\begin{tabular}{|c|c|c|c|c|c|c|c|c|}
\hline \multirow{2}{*}{$\begin{array}{l}\text { Caminhantes } \\
\text { Sexo }\end{array}$} & \multirow[t]{2}{*}{ RP Bruta } & \multicolumn{2}{|c|}{ IC $95 \%$} & \multirow[t]{2}{*}{$\mathrm{p}$} & \multirow[t]{2}{*}{ RP Ajustada } & \multicolumn{2}{|c|}{ IC 95\% } & \multirow[t]{2}{*}{$\mathrm{p}$} \\
\hline & & & & & & & & \\
\hline Feminino & Ref & Ref & Ref & & Ref & Ref & Ref & \\
\hline Masculino & 0,75 & 0,71 & 0,78 & 0,001 & 0,67 & 0,60 & 0,75 & 0,001 \\
\hline \multicolumn{9}{|l|}{ Idade } \\
\hline Crianças/adolescentes ( $0-18$ anos) & Ref & Ref & Ref & & Ref & Ref & Ref & \\
\hline Adultos jovens (19 - 40 anos) & 0,33 & 0,31 & 0,34 & 0,001 & 0,53 & 0,39 & 0,71 & 0,001 \\
\hline Adultos (41 - 64 anos) & 0,37 & 0,35 & 0,40 & 0,001 & 0,51 & 0,38 & 0,70 & 0,001 \\
\hline Idosos (> 65 anos) & 0,54 & 0,50 & 0,60 & 0,001 & 0,55 & 0,39 & 0,77 & 0,001 \\
\hline \multicolumn{9}{|l|}{ Classe econômica } \\
\hline $\mathrm{E}$ & Ref & Ref & Ref & & Ref & Ref & Ref & \\
\hline $\mathrm{D}$ & 0,45 & 0,39 & 0,53 & 0,001 & 0,69 & 0,59 & 0,81 & 0,001 \\
\hline $\mathrm{C}$ & 0,38 & 0,31 & 0,46 & 0,001 & 0,61 & 0,49 & 0,75 & 0,001 \\
\hline $\mathrm{B}$ & 0,14 & 0,02 & 0,97 & 0,047 & 0,28 & 0,04 & 1,90 & 0,201 \\
\hline A & 0,36 & 0,06 & 2,30 & 0,280 & 0,66 & 0,10 & 4,40 & 0,671 \\
\hline \multicolumn{9}{|l|}{ Grau de instrução } \\
\hline Analfabeto/não estudante/>7anos & Ref & Ref & Ref & & Ref & Ref & Ref & \\
\hline Ensino médio completo/incompleto & 0,62 & 0,57 & 0,68 & 0,001 & 0,89 & 0,59 & 0,81 & 0,384 \\
\hline Fundamental I/II Completo/incompleto & 1,00 & 0,96 & 1,10 & 0,330 & 1,00 & 0,80 & 1,30 & 0,624 \\
\hline Superior completo/incompleto/pós-graduação & 0,29 & 0,25 & 0,32 & 0,001 & 0,69 & 0,51 & 0,92 & 0,013 \\
\hline \multicolumn{9}{|l|}{ Condição da atividade } \\
\hline Aposentado/Pensionista & Ref & Ref & Ref & & Ref & Ref & Ref & \\
\hline Dona de casa & 1,50 & 1,40 & 1,70 & 0,001 & 1,20 & 1,00 & 1,40 & 0,046 \\
\hline Em licença & 0,71 & 0,44 & 1,10 & 0,159 & 0,64 & 0,28 & 1,40 & 0,279 \\
\hline Estudante & 1,80 & 1,70 & 2,00 & 0,001 & 0,90 & 0,64 & 1,20 & 0,518 \\
\hline Nunca trabalhou & 0,91 & 0,51 & 1,60 & 0,741 & 0,00 & 0,00 & 1,20 & 0,001 \\
\hline Não ocupado & 1,00 & 0,90 & 1,20 & 0,528 & 1,00 & 0,80 & 1,30 & 0,759 \\
\hline Ocupado & 0,55 & 0,50 & 0,61 & 0,001 & 0,62 & 0,51 & 0,74 & 0,001 \\
\hline Ocupado eventualmente & 1,10 & 0,98 & 1,40 & 0,076 & 1,20 & 0,95 & 1,60 & 0,104 \\
\hline
\end{tabular}

*n $=26.064$. Significância estatística se $\mathrm{p}<0,05 ; \mathrm{RP}$ ajustada segundo o modelo conceitual, incluindo sexo, idade, classe econômica, grau de instrução e condição da atividade; IC 95\% = Intervalo de Confiança 95\%.

ferior ao da Região Metropolitana de São Paulo, que é de aproximadamente 7 vezes $^{18}$. Algumas hipóteses são levantas para explicar a diferença entre os sexos, como menor percepção de segurança, preferência por ciclovias segregadas, além de longas distâncias durante o deslocamento ${ }^{18}$.

Para a classe econômica, encontramos que a Região Metropolitana de Campinas segue o padrão encontrado no Brasil, no qual as classes menos favorecidas são as que mais fazem uso da bicicleta ${ }^{8,12,21}$. O resultado que nos chama mais atenção nessa variável é o de não encontrarmos nenhum ciclista nas classes A/B. Entretanto isso também foi constatado no levantamento "Retratos da sociedade brasileira: locomoção urbana", que encontrou frequências de $0 \%$ de ciclistas que recebem mais de 10 salários mínimos e $1 \%$ naqueles que recebem entre 5 e 10 salários mínimos ${ }^{8}$.

Isso pode estar relacionado ao aspecto cultural presente no Brasil e à herança de como as cidades foram planejadas e estruturadas, para atender e favorecer os interesses de uma pequena parcela da sociedade detentora do capital ${ }^{22}$ que possui mais acesso aos automóveis. Contudo, se olharmos para o nível de atividade física veremos que esse é um dos raros momentos em que as classes menos favorecidas têm um benefício em relação as classes mais favorecidas ${ }^{12}$, entretanto sabemos que a escolha desse modo de transporte não se dá por opção, mas sim por necessidade ${ }^{12}$. 
Em relação aos caminhantes, encontramos associação com as variáveis de sexo e idade. Constatamos que ser mulher teve associação positiva, igualando-se ao padrão apresentado por alguns estudos ${ }^{8,13,23}$. Algumas questões levantadas na literatura podem explicar o motivo disso ocorrer, como o fato das viagens feitas por mulheres serem mais diversificadas em relação aos homens, já que algumas tarefas como fazer compras, levar os filhos à escola, ao médico recaem precipuamente sobre as mulheres ${ }^{24}$ e que a frequência de utilização dos carros para os deslocamentos é maior nos homens ${ }^{23}$.

Quanto à idade, verificou-se associação positiva nas crianças/adolescentes, corroborando os dados encontrados por $\mathrm{Sá}^{12}$. O que pode explicar essa associação é a alta frequência de estudantes, uma vez que famílias e o poder público buscam encontrar escolas próximas da residência das crianças e adolescentes, muitas vezes em uma distância que favorece o deslocamento a pé. Tais achados estão alinhados aos encontrados na Região Metropolitana de São Paulo, os quais indicam que apesar do aumento do transporte privado, a maior parte das crianças e adolescentes se deslocam ativamente para ir à escola $^{25}$. Também encontramos que o principal motivo para o deslocamento das crianças/adolescentes da Região Metropolitana de Campinas foi o estudo regular ${ }^{15}$ e, como indicado na literatura, a maioria são provenientes da escola pública ${ }^{26,27}$.

A variável tempo de viagem é tida como uma das mais importantes, visto que é o principal fator para a escolha do modo de transporte da população brasileira ${ }^{8}$. Na Região Metropolitana de Campinas, houve um aumento no tempo nas viagens de bicicleta e uma queda nas viagens a pé, entretanto os valores encontrados em 2011 não diferem muito do Reino Unido ${ }^{28}$. O tempo de viagens é relativamente curto, visto que normalmente as viagens com deslocamento ativo são realizadas em pequenas distâncias ${ }^{13,21,29}$.

Os residentes dos municípios com população $<100.000$ habitantes exibem as maiores frequências de ciclistas $^{8}$, já para os caminhantes encontramos maiores frequências naqueles com população $>100.000$ habitantes, o município de Campinas apresentou as menores frequências nos dois modos de transporte analisados. Isso pode ocorrer devido à facilidade e maior segurança de se deslocar de bicicleta ou a pé em cidades meno$\mathrm{res}^{30}$. Em todas categorias, houve queda da frequência de viagens de seus residentes, o que já era esperado pela queda na frequência já apontada anteriormente.

Em relação às viagens de bicicleta com integração com outros modais, não encontramos nenhuma viagem com tais características, sendo padrão brasileiro a baixa frequência de integração $(26,4 \%$ de viagens de bicicleta com integração) ${ }^{21}$. Isso pode ocorrer devido à falta de estrutura para proporcionar esse tipo de integração, pois ainda enfrentamos problemas primários com transporte público.

O estudo possui algumas limitações, como o ano da coleta, entretanto avaliamos as duas únicas pesquisas realizadas na região e não encontramos informações sobre uma próxima pesquisa origem e destino a ser realizada. Além disso, informações referentes apenas para um dia útil e o tempo de viagem foi auto relatado, isso pode subestimar ou superestimar os dados, entretanto um diário de viagem era entregue na primeira visita do entrevistador a fim de diminuir esses vieses.

E por fim o fato de não controlarmos a influência que o domicilio possui nos indivíduos, apesar disso criamos diversas categorias que descrevem bem o deslocamento ativo na região e são categorias normalmente utilizadas em outros estudos, o que facilita a comparação. Notou-se também uma dificuldade em encontrar estudos que abordassem exclusivamente o deslocamento a pé em relação aos estudos que abordam somente a bicicleta, no presente estudo analisamos os dois modais separados, a fim de contribuir na literatura de ambos.

Apesar dessas limitações, o presente estudo possui uma amostra representativa da Região Metropolitana de Campinas, a qual localiza-se no interior do estado de São Paulo e foi pouco explorada no aspecto do deslocamento ativo, contribuindo com o aumento de evidências sobre deslocamento ativo e utilizando os seus dois principais modos, lidando com muitas variáveis acerca do tema e podendo fornecer dados para uma futura comparação com as próximas pesquisas Origem e Destino da Região Metropolitana de Campinas.

Ademais, imprime grande importância para o entendimento desse fenômeno estudarmos diferentes regiões do país, a fim de gerar dados para que os órgãos públicos possam conhecer e identificar as necessidades desses municípios.

Em virtude dos dados descritos e analisados, conclui-se que a frequência de ciclistas, caminhantes e suas respectivas viagens diminuiu do ano de 2003 para 2011 e que os principais fatores associados aos ciclistas são sexo masculino e classe econômica mais baixas, já para os caminhantes são sexo feminino e a faixa etária de crianças e adolescentes.

Também foi constatado que o tempo de viagem é 
curto, mesmo com aumento para deslocamentos de bicicleta e diminuição para os realizados a pé, impulsionando a ideia de que sejam rotas de pequenas distâncias. Foi comprovado ainda, que os municípios menores são mais ativos no deslocamento e nenhuma integração entre bicicleta e modos motorizados foi encontrada.

Com isso, pode-se dizer que a Região Metropolitana de Campinas segue o padrão encontrado no Brasil em relação a frequência, distribuição na população e tendência temporal do deslocamento ativo. Avaliar diferentes regiões levanta informações importantes e acrescenta ainda mais na discussão sobre as políticas públicas de mobilidade urbana. Como muitas dessas cidades da Região Metropolitana de Campinas ainda estão em processo de crescimento e urbanização, seria interessante somar estudos, não só descritivos e de levantamentos, mas também experimentais, acerca desse tema na região, a fim de consolidar argumentos para rever o modelo de cidade atual e aspirar um modelo que proporcione uma mobilidade mais ativa e saudável para seus habitantes.

\section{Conflito de interesse}

Os autores declaram não haver conflito de interesse.

\section{Financiamento}

O presente trabalho foi realizado com apoio da Coordenação de Aperfeiçoamento de Pessoal de Nível Superior - Brasil (CAPES) - Código de Financiamento 001.

\section{Contribuição dos autores}

Goulardins GS, participou de todos os processos do estudo, desde a concepção, desenho de estudo, análises estatísticas e escrita do mesmo. Sá TH, participou da elaboração do problema de pesquisa, desenho do estudo e análise dos resultados e escrita do mesmo, coorientou esse estudo. Kokubun E, realizou a orientação do estudo, participando de todas as etapas do estudo.

\section{Agradecimentos}

Os autores agradecem a Universidade Estadual Paulista "Júlio de Mesquita Filho", o departamento de educação física da Unesp de Rio Claro e o programa Ciências da motricidade, além disso agradecemos as empresas responsáveis pela coleta e disponibilização dos dados aos órgãos Secretária dos Transportes Metropolitanos (STM), Companhia do Metropolitano de São Paulo (METRÔ), Empresa Metropolitana de Transportes Urbanos (EMTU), Empresa Paulista de Planejamento Metropolitano S.A (EMPLASA). Além disso, agradecemos as ilustres Beatriz Chritofoletti e Talita Matielo. E por fim a CAPES.

\section{Referências}

1. Ogilvie D, Bull F, Cooper A, Rutter H, Adams E, Brand C, et al. Evaluating the travel, physical activity and carbon impacts of a 'natural experiment' in the provision of new walking and cycling infrastructure: Methods for the core module of the iConnect study. BMJ Open. 2012;2(1):e000694.

2. Sá TH. Como estamos indo? Estudo do deslocamento ativo no Brasil. 2016. 298 f. Tese (Doutorado) - Curso de Nutrição em Saúde Pública, Universidade de São Paulo, São Paulo; 2016.

3. Hamer M, Chida Y. Active commuting and cardiovascular risk: a meta-analytic review. Prev Med. 2008;46(1):9-13.

4. Murtagh EM, Nichols L, Mohammed MA, Holder R, Nevill AM, Murphy MH. The effect of walking on risk factors for cardiovascular disease: An updated systematic review and meta-analysis of randomised control trials. Prev Med. 2015;72:34-43.

5. Furie GL, Desai MM. Active Transportation and Cardiovascular Disease Risk Factors in U.S. Adults. Am J Prev Med. 2012:43(6):621-8.

6. Stevenson M, Thompson J, Sá TH, Ewing R, Mohan D, McClure R, et al. Land use, transport, and population health: estimating the health benefits of compact cities. Lancet. 2016;388(10062):2925-35.

7. Hallal PC, Andersen LB, Bull FC, Guthold R, Haskell W, Ekelund U, et al. Global physical activity levels: surveillance progress, pitfalls, and prospects. Lancet. 2012;380(9838):247-57.

8. Brasil. Andrade RB. Presidente Confederação Nacional da Indústria - Cni (Comp.). Pesquisa CNI - IBOPE. Retratos da sociedade Brasileira: locomoção urbana. Brasília, 2011. Brasília: Cni - Confederação Nacional da Indústria; 2011.

9. Heinen E. Bicycle commuting. IOS Press. Amsterdam; 2011.

10. Sá TH, Rezende LFM, Borges MC, Nakamura PM, Anapolsky S, Parra D, et al. Prevalence of active transportation among adults in Latin America and the Caribbean: a systematic review of population-based studies. Rev Panam Salud Publica. 2017;41:e35:1-11.

11. Kienteka M, Fermino R, Reis R. Fatores individuais e ambientais associados com o uso de bicicleta por adultos: uma revisão sistemática. Rev Bras Ativ Fís Saúde. 2014;19(1):12-24.

12. Sá TH,Pereira RH,Duran AC,Monteiro CA. Socioeconomic and regional differences in active transportation in Brazil. Rev de Saúde Pública. 2016;50(37):1-9.

13. Nakamura PM, Papini CB, Teixeira IP, Fernandes RA, Kokubun E.Associação da caminhada no lazer e no transporte com ambiente construído em adultos do município de Rio Claro-SP. Rev Bras Ativ Fís Saúde. 2013;18(4):424-34.

14. Bauman AE, Reis RS, Sallis JF, Wells JC, Loos RJ, Martin BW, et al. Correlates of physical activity: why are some people physically active and others not?. Lancet. 2012;380(9838):258-71.

15. Goulardins GS. Padrão de deslocamento ativo da Região Metropolitana de Campinas - SP. 101 f. Dissertação (Mestrado) - Curso de Ciências da Motricidade, Educação Física, Universidade Estadual Paulista, Rio Claro; 2019.

16. Biddle SJH, Nigg CR. Theories of exercise behavior. Int J Sport Psychol. 2000;31(2):290-304.

17. Dumith SC. Proposta de um modelo teórico para a adoção da prática de atividade física. Rev Bras Ativ Fís Saúde. 2008;13(2):110-20.

18. Sá TH, Duran AC, Tainio M, Monteiro CA, Woodcock J. Cycling in São Paulo, Brazil (1997-2012): Correlates, time trends and health consequences. Prev Med Rep. 2016;13(4):540-5. 
19. Presidência da República. Decreto número 7222, de 29 de junho de 2010. Altera a Tabela de Incidência do Imposto sobre Produtos Industrializados-TIPI, aprovada pelo Decreto número 6006, de 28 de dezembro de 2006. Seção 1-edição extra; 122. Diário Oficial da União. 2010.

20. Campos VB, Borges IC. Estudo Temático Área: Sustentabilidade: evolução da frota de veículos na região metropolitana de campinas no período de 2001 a 2018. Evolução da Frota de Veículos na Região Metropolitana de Campinas no período de 2001 a 2018.2019.

21. Brasil. Transporte Ativo. Perfil do Ciclista Brasileiro. 2015.

22. Sá TH, Monteiro CA. Deslocamento ativo, mobilidade urbana e saúde. In: Rodrigues GS. A cidade em equilíbrio: Contribuições teóricas ao 3 o. Fórum Mundial da Bicicleta Curitiba. 2014;3(1):231-6.

23. Teixeira IP, Nakamura PM, Kokubun E. Prática de caminhada no lazer e no deslocamento e associação com fatores socioeconômicos e ambiente percebido em adultos. Rev Bras Cineantropom Desempenho Hum. 2014;16(3):345-58.

24. Svab, Haydée. Evolução dos padrões de deslocamento por gênero: Um estudo da Região Metropolitana de São Paulo. 472 f Dissertação (Mestrado). Universidade de São Paulo, São Paulo. 2016.

25. Sá TH, Garcia LMT, Mielke GI, Rabacow FM, Rezende LFM. Changes in travel to school patterns among children and adolescents in the São Paulo Metropolitan Area, Brazil, 1997-2007. J Transp Health. 2015;2(2):143-50.
26. Silva KS, Garcia L, Oliveira ESA, Duca GFD, Araújo VC, Nahas MV. Fatores associados ao deslocamento ativo para o trabalho em industriários da Paraíba. J Phys Educ. 2011;22(2):265-72.

27. Rech RR, Rosa CO, Avrela PR, Halpern R, Constanzi CB, Bergmann MLA, et al. Fatores associados ao deslocamento ativo em escolares. Rev Bras Ativ Fís Saúde. 2013;18(3):332-8.

28. National Travel Survey statistics (Org.). National Travel Survey: 2017. 2018. Disponível em: https://www.gov.uk/ government/statistics/national-travel-survey-2017.

29. Gomes GA, Reis RS, Parra DC, Ribeiro I, Hino AA, Hallal PC, et al. Walking for leisure among adults from three Brazilian cities and its association with perceived environment attributes and personal factors. Int J Behav Nutr Phys Act. 2011;8(111).

30. Teixeira IP. Impacto da implementação de ciclofaixas na utilização da bicicleta como meio de transporte. $201 \mathrm{f}$. Tese (Doutorado) - Curso de Ciências da Motricidade, Educação Física, Universidade Estadual Paulista, Rio Claro; 2017.

Recebido: 01/07/2020 Aprovado: 10/05/2021 